\title{
LANGUAGE TYPOLOGY AND UNIVERSALS
}

\section{STUF - SPRACHTYPOLOGIE UND UNIVERSALIENFORSCHUNG}

EDITOR-IN-CHIEF

Thomas Stolz, Bremen

EXECUTIVE EDITOR

Cornelia Stroh, Bremen

EDITORIAL BOARD

Walter Bisang, Mainz

William Croft, Albuquerque, N.M.

Wolfgang $U$. Dressler, Wien

Ray Fabri, Malta

José Antonio Flores Farfán, Mexico DF

Lutz Gunkel, Mannheim

Stefanie Haberzettl, Saarbrücken

Bernhard Hurch, Graz

Maria Koptjevskaja-Tamm, Stockholm

Susanne Michaelis, Leipzig

Marianne Mithun, Santa Barbara, C.A.

Claire Moyse-Faurie, Villejuif/Paris

Damaris Nübling, Mainz

Ignazio Putzu, Cagliari

Wolfgang Raible, Freiburg

Jeanette Sakel, Bristol

Christoph Schroeder, Potsdam

Eva Schultze-Berndt, Manchester

Christel Stolz, Bremen

Elisabeth Verhoeven, Berlin

\section{DE GRUYTER \\ MOUTON}


ABSTRACTED/INDEXED IN Baidu Scholar · Cabell's Directory · CNKI Scholar (China National Knowledge Infrastructure) - CNPIEC: cnpLINKer - Dimensions · EBSCO (relevant databases) . EBSCO Discovery Service · ERIH PLUS (European Reference Index for the Humanities and Social Sciences) · Genamics JournalSeek · Google Scholar · IBR (International Bibliography of Reviews of Scholarly Literature in the Humanities and Social Sciences) · IBZ (International Bibliography of Periodical Literature in the Humanities and Social Sciences) · J-Gate · JournalTOCs · KESLI-NDSL (Korean National Discovery for Science Leaders) · Linguistic Bibliography · Linguistics Abstracts Online $\cdot$ Microsoft Academic $\cdot$ MLA International Bibliography $\cdot$ Naviga (Softweco) $\cdot$ Primo Central (ExLibris) · ProQuest (relevant databases) · Publons · QOAM (Quality Open Access Market) ·

ReadCube · SCImago (SJR) · SCOPUS · Summon (Serials Solutions/ProQuest) · TDNet · Ulrich's Periodicals Directory/ulrichsweb · WanFang Data - Web of Science: Emerging Sources Citation Index - WorldCat (OCLC)

ISSN 1867-8319 · e-ISSN 2196-7148

All information regarding notes for contributors, subscriptions, Open access, back volumes and orders is available online at www.degruyter.com/journals/stuf

RESPONSIBLE EDITOR Prof. Dr. Thomas Stolz, Universität Bremen, Fachbereich 10, Postfach 330440, 28334 Bremen, Germany, Email: stolz@uni-bremen.de

EXECUTIVE EDITOR Dr. Cornelia Stroh, Universität Bremen, Fachbereich 10, Postfach 330440, 28334 Bremen, Germany, Email: cornelia.stroh@uni-bremen.de

JOURNAL MANAGER Anna Barkhoff, De Gruyter, Genthiner Straße 13, 10785 Berlin, Germany, Tel.: +49 (0)30 260 05-317, Fax: +49 (0)30 260 05-250, Email: anna.barkhoff@ degruyter.com

RESPONSIBLE FOR ADVERTISEMENTS Claudia Neumann, De Gruyter, Genthiner Straße 13, 10785 Berlin, Germany, Tel.: +49 (0)30 260 05-226, Fax: +49 (0)30 260 05-264,

Email: anzeigen@degruyter.com

(C) 2019 Walter de Gruyter GmbH, Berlin/Boston

TYPESETTING Integra Software Services Pvt. Ltd., Pondicherry, India

PRINTING Franz X. Stückle Druck und Verlag e.K., Ettenheim

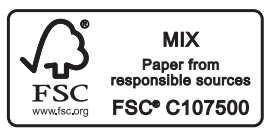




\section{Contents}

Michael Daniel

Bagvalal place names as adverbs -297

Martin Haspelmath

Differential place marking and differential object marking — 313

Julia Nintemann and Maja Robbers

Towards a typology of spatial deictic expressions - 335

Thomas Stolz and Nataliya Levkovych

Absence of material exponence - 373

Axel Holvoet

On the heterogeneity of deaccusative reflexives — 401

One-Soon Her, Marc Tang and Bing-Tsiong Li

Word order of numeral classifiers and numeral bases -421 